\title{
Time-resolved imaging of compressible air-disc under a drop impacting on a solid surface
}

\author{
By E. Q. Li and S. T. Thoroddsen \\ Division of Physical Sciences and Engineering and Clean Combustion Research Center, \\ King Abdullah University of Science and Technology (KAUST), \\ Thuwal, 23955-6900, Saudi Arabia.
}

(Received 5 August 2015)

When a drop impacts on a solid surface, its rapid deceleration is cushioned by a thin layer of air, which leads to the entrapment of a bubble under its center. For large impact velocities the lubrication pressure in this air layer becomes large enough to compress the air. Herein we use highspeed interferometry, with 200 ns time-resolution, to directly observe the thickness evolution of the air-layer during the entire bubble entrapment process. The initial disc radius and thickness shows excellent agreement with available theoretical models, based on adiabatic compression. For the largest impact velocities the air is compressed by as much as a factor of 14 . Immediately following the contact, the air disc shows rapid vertical expansion. The radial speed of the surface minima just before contact, can reach 50 times the impact velocity of the drop.

\section{Introduction}

The capture of a bubble under an impacting drop can have detrimental effects on the uniformity of spray coatings and interfere with precision inkjet-based manufacturing, like the printing of organic displays. For the lowest impact velocities or superhydrophobic surfaces, the air cushion can allow rebounding of the drop and prevent molecular contact with the substrate (Richard et al. (2002), de Ruiter et al. (2012), van der Veen et al. (2012), Kolinski et al. (2012)). However, above a modest impact velocity the drop always makes contact along a ring, entrapping a central bubble. Early snapshots of these bubbles are due to Chandra \& Avedisian (1991) and Thoroddsen \& Sakakibara (1998), but without any dynamical details. Van Dam \& Le Clerc (2004) also observed bubbles under small inkjet droplets. Thoroddsen et al. (2005) used high-speed video imaging to capture the air-disc formation and its contraction into a central bubble. They also observed pinchoff of a micro-droplet inside the entrapped bubble. This occurs when capillary waves converge to the axis of symmetry and their apex touches the substrate. Lee et al. (2012) used X-ray imaging to confirm these dynamics in renewed detail.

Previous time-resolved interferometric measurements of the air-layer shape under the drop, have been limited to very low impact velocities $(\leq 0.22 \mathrm{~m} / \mathrm{s})$ mostly in the bouncing regime. These are due to de Ruiter et al. (2012) who used a two-color setup and by van der Veen et al. (2012) who used white-light color interferomentry. Kolinski et al. (2012) used a total internal reflection (TIR) setup, to characterize the closest approach of the drop to the substrate. The focus has been either on the incompressible bouncing dynamics, which can occur even for hydrophilic surfaces (de Ruiter et al. (2014), Kolinski et al. (2014)), or on predicting the size of the entrapped bubble. Bouwhuis et al. (2012) showed there exists a maximum in the entrapped bubble size for a fairly low impact velocity. Klaseboer et al. (2014) formulated a unifying theory for drops entrapping bubbles, proposing a slightly different powerlaw, based on a different velocity potential within the drop.

Driscoll \& Nagel (2011) used interferometry to image the central air disc for drop impacts under a reduced pressure environment and showed that it becomes flatter. Liu et al. (2013) have recently reported the first interference imaging under similar impact conditions as herein. However, the imaging in these studies were not time-resolved.

Theoretical modeling of the air cushioning under the drop was started with the 2-D theories of Smith et al. (2003) and Korobkin et al. (2008). These are based on the lubrication approximation for the air-flow within the thin film, while using inviscid potential theory for the deformations 
(a)

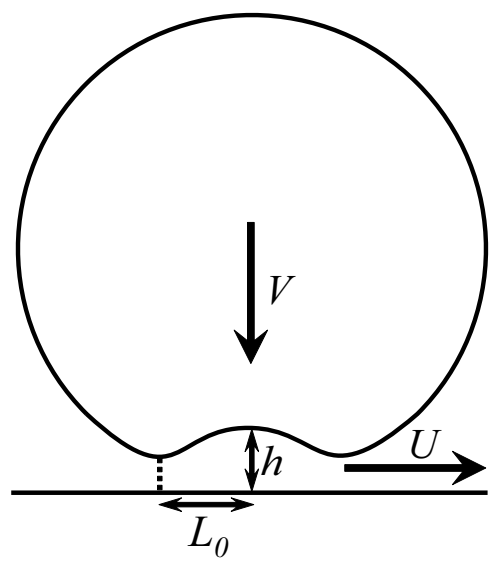

(b)

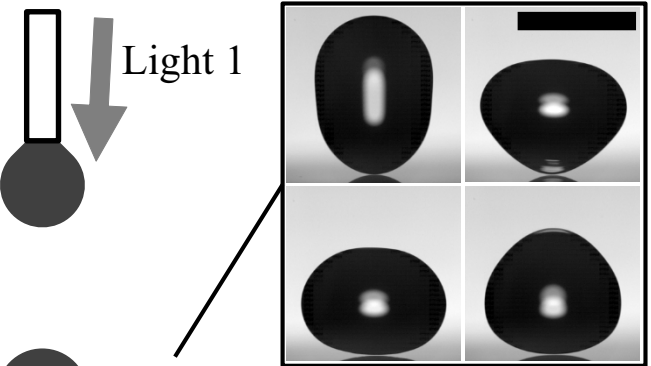

Droplet

Glass slide

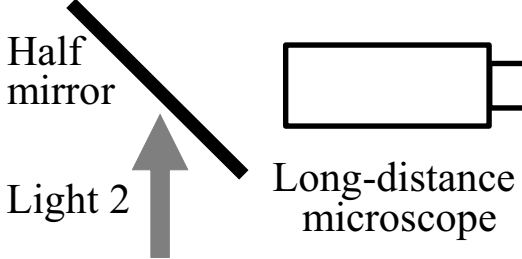

FiguRE 1. Experimental setup. (a) Definition of the radial width $L_{o}$ and center-line height $h$ of the air-layer. (b) Optical setup and a range of drop shapes (Scale bar is $5 \mathrm{~mm}$ ). We use either lighting from below with a half-silvered pellicle beam splitter, or a solid bottom mirror, with lighting from the top going through the drop. Drop shapes are shown at $t=86 \mathrm{~ms}$ (top left); $106 \mathrm{~ms}$ (top right); $111 \mathrm{~ms}$ (bottom left) \& 116 ms (bottom right) after pinch-off from the nozzle.

within the drop. Later, Hicks \& Purvis (2010) reformulated the theory for the 3-D axisymmetric configuration, which allows for a more direct comparison with experiments.

Mandre, Mani \& Brenner (2009) expanded the lubrication theory to allow for compression of the gas, when the impact velocity becomes large enough. They used the ideal gas law to predict the minimum air-disc thickness for either isothermal or adiabatic gas compression. Their subsequent work used the same equation of state with various values of the ratio of the specific heats $\gamma$ to describe drop skating on a thin layer of air (Mani et al. (2010)) and then focused on the isothermal case, to study the related precursors of splashing (Mandre \& Brenner (2012)). Hicks \& Purvis (2013) followed up the studies of gas compression, with full analysis of the energy conservation in the gas. They further tested their theories by comparison with large-scale experiments on the impact of spherical solid sections (Hicks et al. (2012)).

Herein we exploit the latest high-speed video technology to observe the air entrapment at about two-orders of magnitude faster frame-rates than in earlier observations (de Ruiter et al. (2012), van der Veen et al. (2012), Kolinski et al. (2012), Driscoll \& Nagel (2011), Liu et al. (2013)). This allows us, for the first time, to obtain time-resolved interface shapes over the entire process, for high impact velocities well into the regime where gas compressibility is important (Mandre et al. (2009)).

\section{Experimental Setup}

Our imaging is made possible by the Kirana-05M ultra-high-speed video camera (Specialised imaging, Tring, UK), see Crooks et al. (2013), which is capable of capturing 180 frames at up to 5 million frames-per-second (Mfps). The sensor has $910 \times 764$ active pixels, irrespective of the frame-rate. Triggering is accomplished by the falling drop cutting the view of a horizontal linesensor (SI-OT3). The optical setup is sketched in Fig. 1(b). In combination with a long-distance microscope (Leica Z16 APO) at magnification of 29.5 we achieve a spatial resolution of $1.03 \mu \mathrm{m} / \mathrm{px}$, even at the highest frame-rates. We use 1-mm-thick microscope glass slide (Corning micro-slides $2947-75 \times 50)$ as a substrate. The rms roughness measured with AFM is of the order of $3 \mathrm{~nm}$, with largest asperities around $10 \mathrm{~nm}$. The impact is viewed from below, through the glass slide, with columnated illumination coming either through the drop, or through a bottom beam-splitter. Top lighting gives clearer images of the initial contact line. To minimize angular deflection of the columnated beam, we align it close to the vertical. For illumination we use 180 pulsed diode lasers 


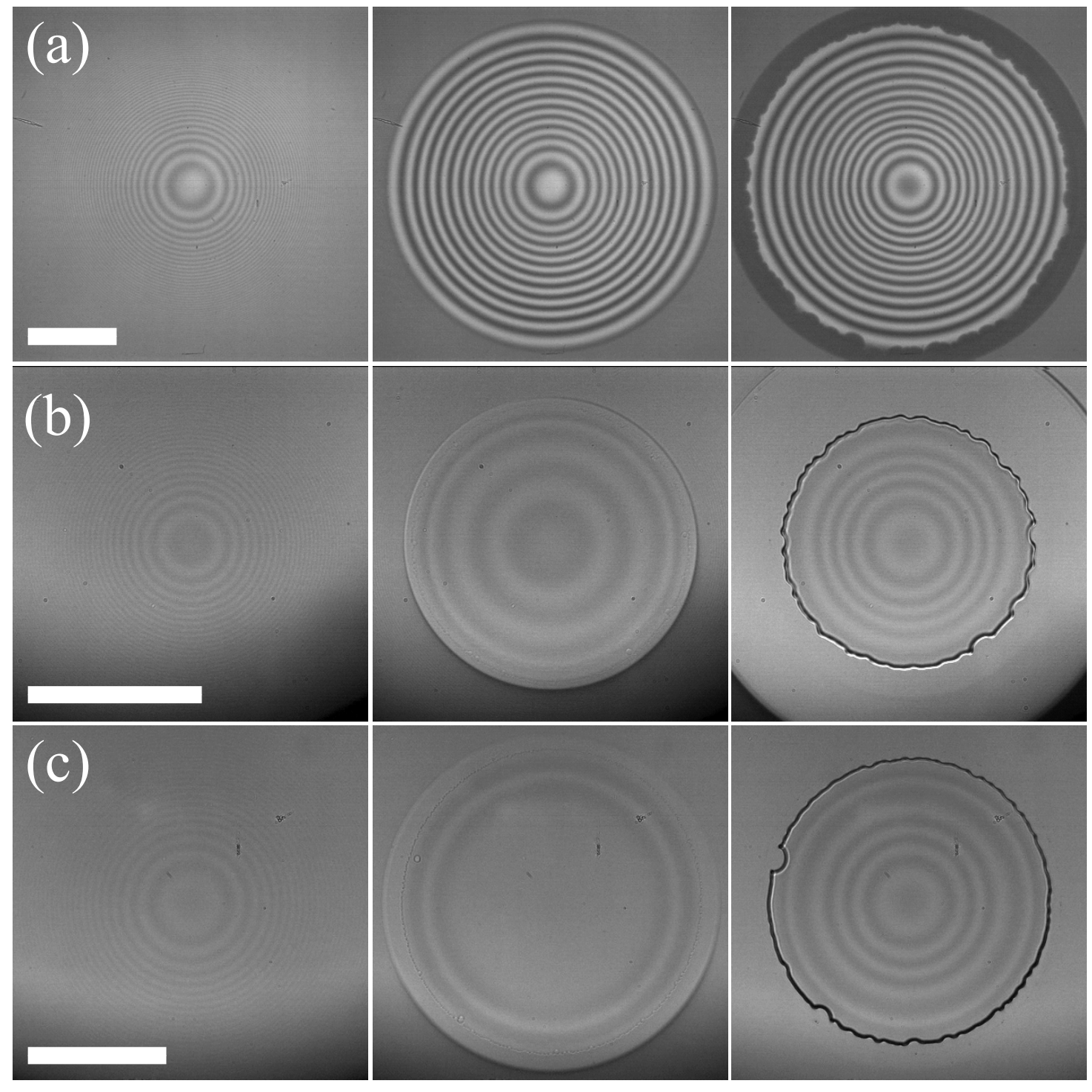

Figure 2. The observed interference fringes taken from $5 \mathrm{Mfps}$ video clips. (a) For impact velocity $V=$ $1.06 \mathrm{~m} / \mathrm{s}, R_{b}=4.1 \mathrm{~mm}$. Times shown at $t=-15.6,0$ and $2.2 \mu$ s relative to first contact along the ring. Lighting from bottom. (b) Fringes for $V=2.53 \mathrm{~m} / \mathrm{s}, R_{b}=1.6 \mathrm{~mm}$, at $t=-3.8,0$ and $2.2 \mu \mathrm{s}$ from first contact. Lighting from top. (c) Fringes for $V=4.05 \mathrm{~m} / \mathrm{s}, R_{b}=3.2 \mathrm{~mm}$, at $t=-1.6,0$ and $2 \mu \mathrm{s}$ from first contact. Lighting from top. The middle column shows in all cases the frame closest to first contact, whereas the last panels in each row show the first maximum expansion at the centerline. Scale bars are all $200 \mu \mathrm{m}$ long. See also online movies [Ref.].

(SI-LUX640) with monochrome light, with $\lambda=640 \mathrm{~nm}$ wavelength. This gives us depth resolution, or spacing between a bright and dark fringe, of $\lambda / 4=160 \mathrm{~nm}$. The laser pulses can be synchronized with the framing and their duration controlled down to $50 \mathrm{~ns}$, thereby freezing the motion of the fringes. The $200 \mathrm{~ns}$ interframe time, allows us to follow the individual fringes and thereby extract the shape evolution with time from the initial drop deformation through the contact with the solid surface and during the subsequent decompression and contraction of the entrapped air disc. This removes some of the ambiguity in tracking the absolute layer thickness, without applying the more sophisticated colour interferometry techniques employed by Mugele's and Lohse's research groups (de Ruiter et al. (2012), van der Veen et al. (2012)).

We use large water drops to increase the space- and time-scales of the impact, thereby extracting the best resolution from the instrumentation. This is characterized by a global Reynolds number, $R e=\rho_{l} R V / \mu_{l} \sim 2600-15700$ and Weber number $W e=\rho_{l} R V^{2} / \sigma \sim 36-1300$, where $R$ is the equivalent spherical drop radius $(2.6 \mathrm{~mm})$ and $V$ is the impact velocity $(\sim 1-6 \mathrm{~m} / \mathrm{s}) ; \rho_{l}, \mu_{l}$ and 
$\sigma$ are respectively the density, dynamic viscosity and surface tension of the liquid. Equivalently, a subscript $g$ is used to indicate gas properties.

\section{Results}

\subsection{Shape-evolution of the air disc}

Figure 2 shows examples of the interference fringes for different impact velocities and drop sizes. Figure 2(a) uses bottom reflection interferometry, which gives us better image contrast, as compared to top transmission-light interference fringes shown in Figs. 2(b,c). Furthermore, the liquid-solid contact zone in the right-most panel of Fig. 2(a) appears dark as most of light passes directly through this liquid-solid interface and shines away from the camera. On the other hand, this contact zone is bright in Fig. 2(b,c) as the light passes through the liquid-solid interface towards the camera. The wiggled circular lines in the right-most column represent the location of the inner contact lines at the edge of the enclosed air disk. The smooth circular lines in the upper right corners of $(\mathrm{a}, \mathrm{b})$ are the outer edges of the wet region.

Keep in mind that we use the bottom radius of curvature $R_{b}$, as the characteristic drop dimension, to account for the large shape oscillations following the release from the nozzle, as has been highlighted in other impact studies (Thoroddsen et al. (2005), Hicks \& Purvis (2010), Liu et al. (2013), Thoraval et al. (2013), Wang et al. (2013)). The shape at impact was monitored from side-view, with a separate high-speed video camera (Phantom V1610), at $20 \mathrm{kfps}$ and with a 3 $\mu$ s exposure. Figure 1(b) shows some of the varied shapes of drops studied. The fringes remain axisymmetric, both for top and bottom illumination. Note that for the largest $V$, at first contact there are much fewer fringes, which indicates a thinner air disc.

Figure 3 shows the surface shapes, extracted from the fringes, when the drop approaches, deforms and contacts the glass substrate. The deceleration of the drop bottom is very abrupt, the drop retaining at least $80 \%$ of $V$ to within $10 \mu \mathrm{m}$ from the solid surface. The measured deceleration of the bottom of the drop for $V=4.05 \mathrm{~m} / \mathrm{s}$ is $\simeq 3 \times 10^{5} \mathrm{~g}$. Then the motion at the axis of symmetry reverses and a dimple forms under its center, which is bounded at the outer edge by a kink in the surface, highlighted schematically in Fig. 1(a). The kink moves radially outwards at rapid horizontal velocity $U$. This kink makes the first circular contact with the solid, thereby entrapping the air disc (Korobkin et al. (2008), Mandre et al. (2009), Duchemin \& Josserand (2011), Hicks \& Purvis (2013)). From the video clips we can measure $U$. In Fig. 4 we plot this velocity normalized by the drop impact velocity. For the largest impact velocity, $V=6 \mathrm{~m} / \mathrm{s}$, we get $U / V \simeq 50$, which is even larger than the velocity of the ejecta moving along the substrate, see Thoroddsen et al. (2012).

Mandre et al. (2009) found a self-similar solution of the simplified equations, for the shape and radial velocity of this kink at the outer edge of the dimpled region. This solution makes contact with the solid surface in finite time and the horizontal velocity of the kink location is dominated by radial advection within the liquid. The similarity gives the scaling $U \sim V\left(h_{m i n} / R\right)^{-1 / 2}$, where $h_{\text {min }}$ is the minimum gap under the kink, while $h_{m i n} / R \sim S t^{2 / 3}$ resulting in

$$
U \sim V S t^{-1 / 3}
$$

with a prefactor of $O(1)$. Here $S t$ is defined as the inverse of the conventional Stokes number, i.e.

$$
S t=\frac{\mu_{g}}{\rho_{l} V R_{b}} .
$$

Our data in Fig. 5 shows good correspondence with this scaling with a prefactor of 0.42 . Mandre \& Brenner (2012) (their figure 3) show same power-law with a similar prefactor of 0.34 for the 2-D case, at lower impact velocities. See also the axisymmetric simulations of the surface shapes by Duchemin \& Josserand (2011).

The shape evolution shown in Fig. 3(b,f) is very similar to those obtained from solutions of the lubrication theory, see for example fig. 2 in Duchemin \& Josserand (2011) and fig. 2(a) in Hicks \& Purvis (2013).

The subsequent contraction of the air disc, after liquid-solid contact, into a bubble was successfully modeled by Thoroddsen et al. (2005) assuming an increasing but uniform disc thickness. 

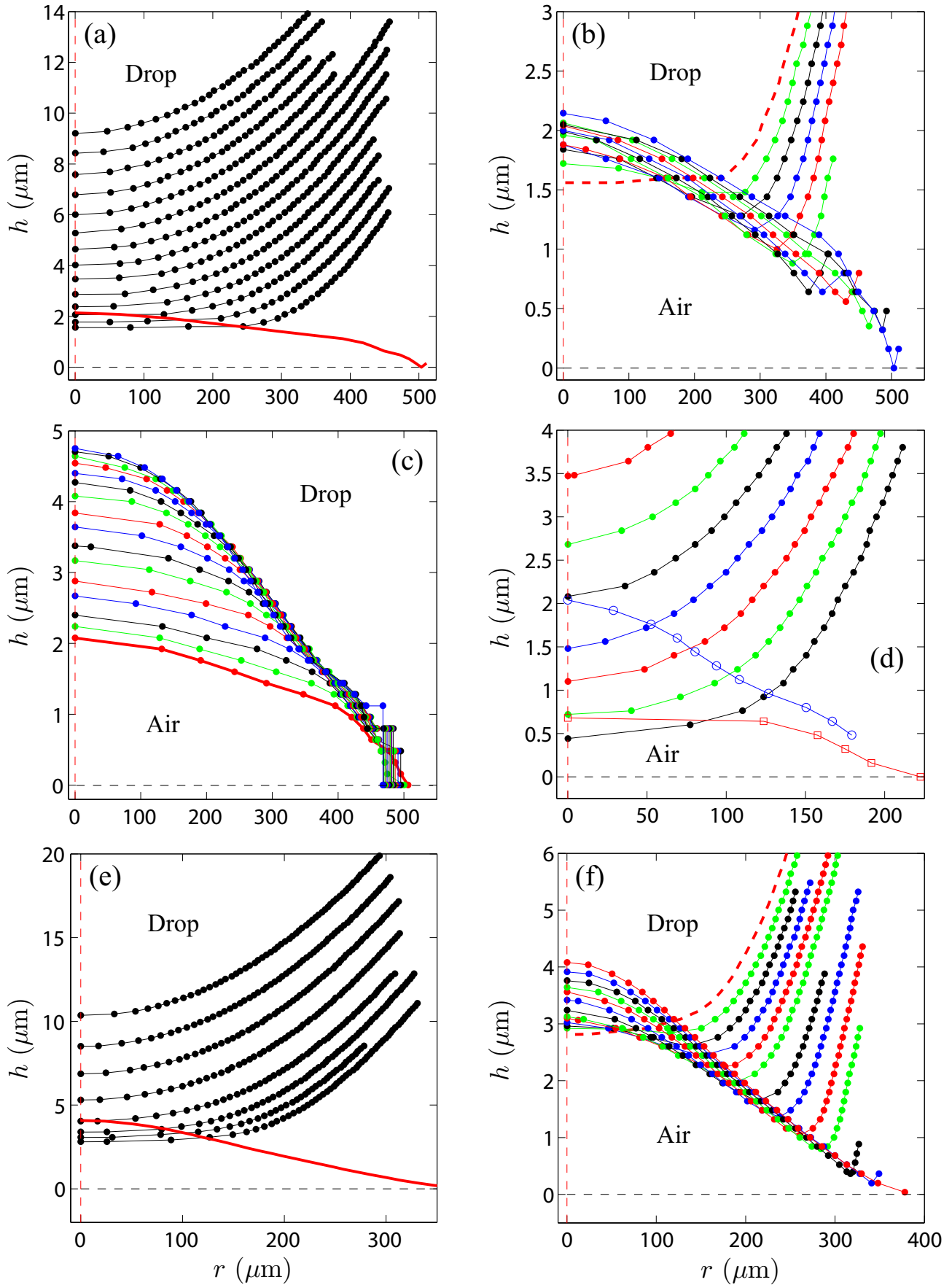

Figure 3. (color online) The air-layer thickness profiles under the drops, measured from the interferometric video clips at $5 \mathrm{Mfps}$. The black dashed horizontal line marks the surface of the solid glass substrate. (a-c) For $V=2.43 \mathrm{~m} / \mathrm{s}$ and $R_{b}=8.2 \mathrm{~mm}\left(\epsilon^{-1}=6.0, S t=9.2 \times 10^{-7}\right)$. (a) The bottom deformation of the approaching drop. The black curves are spaced by $400 \mathrm{~ns}$, with the bottom curve at $2.2 \mu$ s before touchdown, which is shown by the red curve. The dots mark the center locations of the dark or bright fringes, with interpolations at the centerline and at the tip of the downwards kink in (b,f). (b) Detail of the formation of the bottom dimple and touchdown of the outer kink, to entrap the air disc. Note the different vertical scales. The dashed red curve denotes the last profile in (a). Profiles spaced by 200 ns. (c) Rapid expansion of the air-disc, after first contact. Profiles spaced by $400 \mathrm{~ns}$. The thickness of the edge of the contracting air disc can not be measured and we simply draw it as a flat segment, but it is much thicker than the disc, see Thoroddsen et al. (2005), Lee et al. (2012) and Liu et al. (2013). (d) Shapes for $V=4.05 \mathrm{~m} / \mathrm{s}$ and $R_{b}=3.2 \mathrm{~mm}\left(\epsilon^{-1}=14.4, S t=1.4 \times 10^{-6}\right)$. Profiles shown at $t=-2,-1.8,-1.6,-1.4,-1.2$, $-1,-0.8,0,2.2 \mu$ s relative to first contact. In this case the last three profiles before contact (not shown) cannot be determined accurately. The open red squares show the shape of the air disc at first contact and the open blue circles at the end of the rapid expansion. (e-f) Shapes for $V=1.06 \mathrm{~m} / \mathrm{s}$ and $R_{b}=4.1 \mathrm{~mm}$ $\left(\epsilon^{-1}=0.69, S t=4.3 \times 10^{-6}\right)$. (e) Bottom deformation of the drop, at $t=-22,-20,-18,-16,-14,-12.6,-11$ $\&-10.4 \mu$ s before contact (red curve). (f) Closeup of central dimple and downwards kink. Times shown at $t=-10.4,-9.8,-9.2,-8.6,-8,-7.4,-6.4,-5.6,-4.6-3.8,-2.8,-1.6 \& 0 \mu$ s relative to first contact (red curve). 


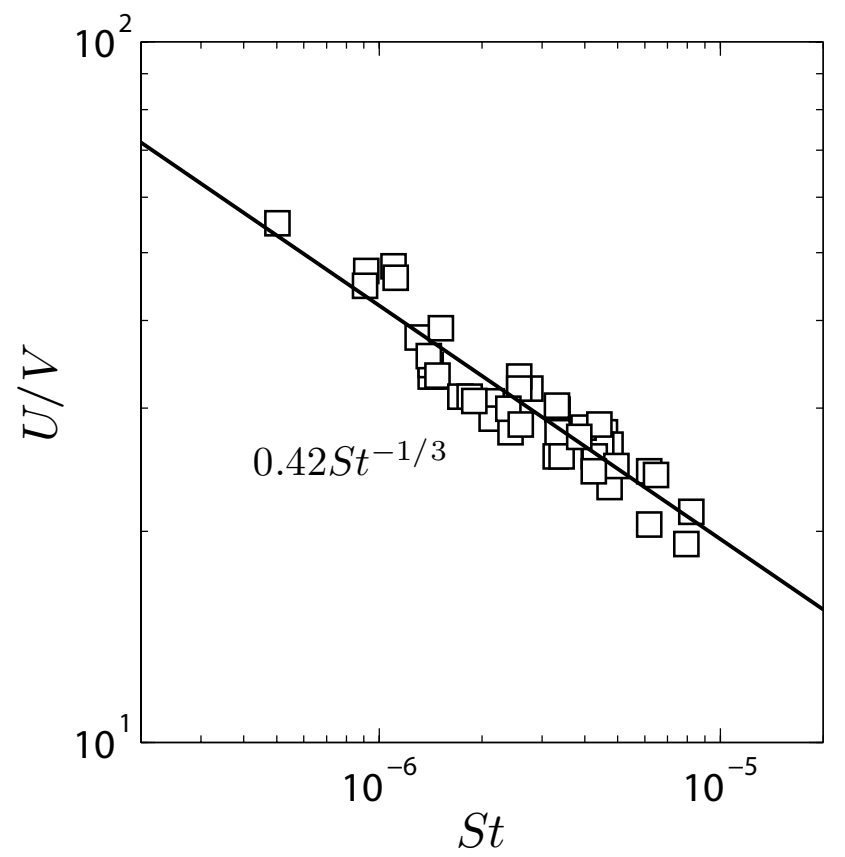

Figure 4. The radial velocity of the minimum thickness kink in the bottom surface, which first touches the substrate, compared with the theoretically predicted power-law from Eq. (3.1). The data encompasses the entire range of impact velocities, including the compressible regime.

However, the edge of the contracting air-disc grows in size forming a rim. Liu et al. (2013) improved upon this model by taking into account this thickening of the edge.

\subsection{Size of the initial air disc}

The deformation of the bottom of the drop, leading to the dimple formation and entrapment of the air-disc, is governed by a balance between the lubrication pressure in the air trying to escape the oncoming drop $P \sim \mu_{g} V R_{b} / H^{2}$, and the inertia of the drop, which must be decelerated before contact, $\rho_{l} V^{2} / H^{*}$, where $H^{*}$ is the thickness of the air layer at the start of drop deformation. This gives a layer height $H^{*} \sim R_{b} S t^{2 / 3}$. Based on this, one can use simple geometric arguments for the horizontal size of the air-disc $\left(L=\sqrt{R_{b} H}\right.$, Mandre et al. (2009)) to get $L / R_{b} \sim S t^{1 / 3}$, in the incompressible case. However, the horizontal extent of the air-disc, where the drop contacts the solid, is determined by the touchdown of the kink in the free surface, described by the above-mentioned similarity solution from Mandre et al. (2009). The drop liquid deforms inviscidly funneling liquid away from the central stagnation point, thereby increasing the velocity components of the kink, which in turn is resisted from contact by the increased pressure in the air layer. This is repeated on ever smaller length-scales until contact is made in finite time, see discussion in Mandre \& Brenner (2012).

Theoreticians prefer to tackle the 2-D configuration (Mandre et al. (2009), Hicks \& Purvis (2013)), representing cylindrical drops. While their scaling arguments carry over from 2-D, only the axisymmetric theory allows quantitative comparison to experiments. Hicks \& Purvis (2010) formulated such a theory for the incompressible case, where they predicted the initial contact radius of the disc,

$$
L_{o}=3.8\left(\frac{4 \mu_{g}}{\rho_{\ell} V}\right)^{1 / 3} R_{b}^{2 / 3} .
$$

Hicks et al. (2012) showed this to be in reasonable agreement with impacting solid spheres and the available droplet data of Thoroddsen et al. (2005), without any adjustable constants. However, while their compressible theory (Hicks \& Purvis (2013)) is limited to 2-D, they have also shown that $L_{o}$ is not dependent on the gas compressibility. This is indeed born out by our data, in Fig. 5 , which shows a perfect fit of $L_{o}$ to their expression, over a large range of impact values. This contradicts earlier results by Liu et al. (2013), where smaller water drops were used and $L_{o}$ was essentially independent of $V$. 


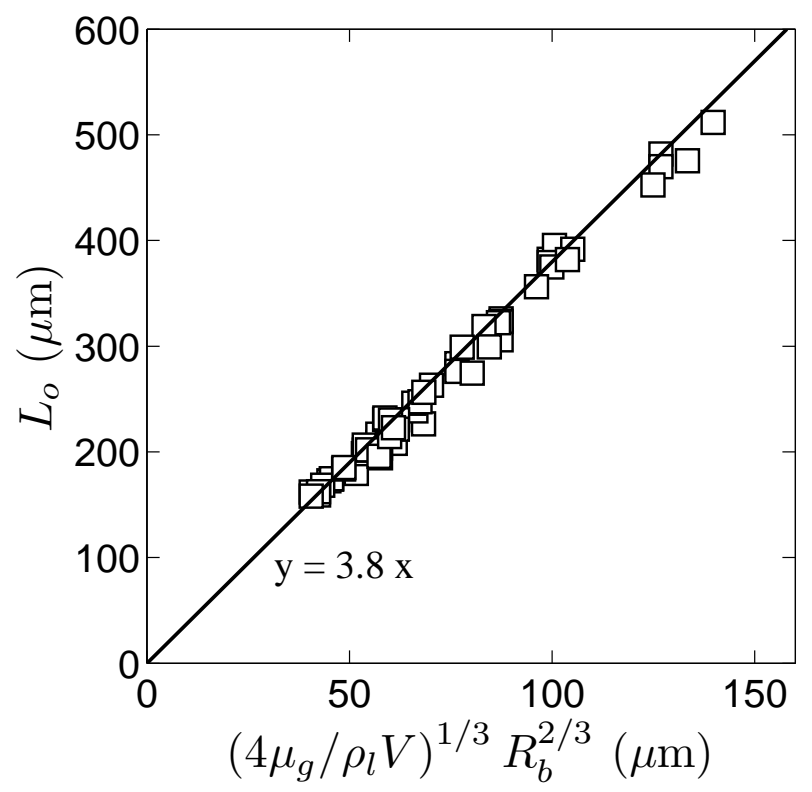

Figure 5. The size of the initial air-disc, measured at first contact with the wall, vs Eq. (3.2), which is based on the theory of Hicks et al. (2012). $R_{b}$ is the bottom radius of curvature of the drop.

Hicks \& Purvis (2013) mention that the adiabatic case of Mandre et al. (2009) predicts that $L_{o}$ should decrease when $\epsilon^{-1}$ increases. Teasing out these subtle differences will require future experiments at reduced pressure.

\subsection{Thickness of the disc and compression of the gas}

Building on previous incompressible theory (Smith et al. (2003), Korobkin et al. (2008)), Mandre, Mani \& Brenner (2009) derived conditions for when compressibility of the gas will come into play for higher impact velocities. This formulation compares the static atmospheric pressure to the lubrication pressure, the ratio of which forms a compressibility factor

$$
\epsilon=\frac{P_{a t m}}{\left(R V^{7} \rho_{\ell}^{4} / \mu_{g}\right)^{1 / 3}} .
$$

If $\epsilon^{-1}>3$ one expects significant compression of the air-disc and thereby smaller values of $H^{*}$. According to Mandre et al. (2009), when using adiabatic compression, the normalized thinning should scale with the compressibility factor as $H^{*} /\left(R_{b} S t^{2 / 3}\right)=3.2 \epsilon^{1 / 3}$. For isothermal compression the power-law is different, i.e. $\sim \epsilon^{1}$. Our values of $\epsilon^{-1}$ reach $\sim 40$ for the large impact velocities $V$ and large bottom radii of curvature $R_{b}$, which takes us well into the compressible regime.

In Fig. 6 we plot the air-layer height at the centerline $h=H^{*}$, at the instant of first contact. Note that our definition of $H^{*}$, taken at first contact, differs from that used in Mandre et al. (2009) and Smith et al. (2003), where they look at $H^{*}$ at the onset of deformation. The two should only differ by a small factor, but our definition is much easier to quantify experimentally. The data fits the theory and numerics of Mandre et al. (2009) reasonably close, when we use their adiabatic case, $\gamma=1.4$ (see their figure 2). Our incompressible asymptote is at 3.4 vs 4.3 in their 2-D case. Our measurements show that the thinning of the air-layer, as compressibility sets in, follows an empirical power-law of $4.2 \epsilon^{0.40}$. This mirrors closely their numerical results when using the ratio of specific heats $\gamma=1.4$, for an adiabatic process, where they see essentially the same exponent of 0.40. This is significantly steeper than their theoretical prediction of an asymptote of $\epsilon^{1 / 3}$, which may be realized at much larger $\epsilon^{-1}$ than in our experiments. Here again our prefactor differs from the value of 3.2 observed in their 2-D case. Liu et al. (2013) observed a much smaller exponent for their impacts of water drops. Note that our results are far from the isothermal $(\gamma=1)$ power-law $\sim \epsilon^{1}$, which is also drawn in the figure.

Selecting the instant of first contact will not show the minimum value of $H^{*}$, as Fig. 3(b,d,f) show the height at the center has rebounded somewhat from its minimum. This is also seen in theoretical studies (Mandre et al. (2009), Duchemin \& Josserand (2011), Hicks \& Purvis (2013)). 


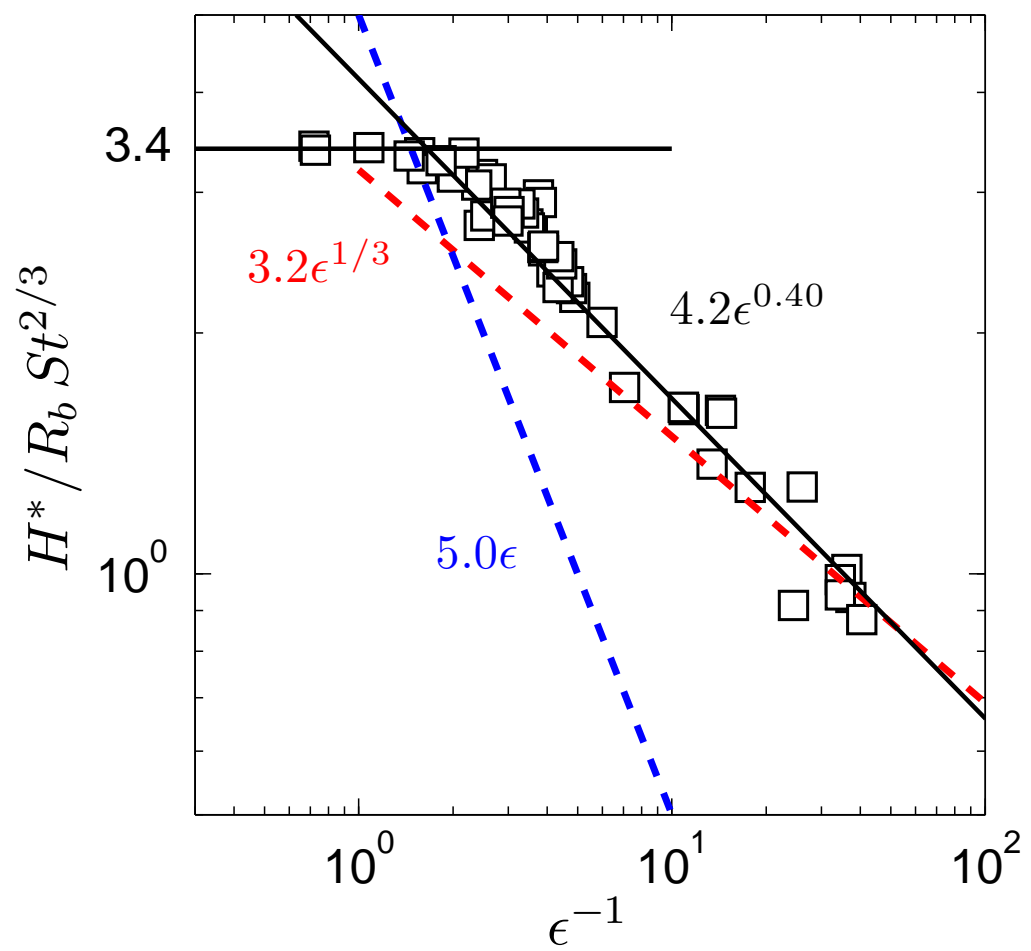

FiguRE 6. The air-film thickness at the centerline, measured at the instant of contact along the outer ring, vs the compressibility parameter in Eq. (3.3). Compared to the theory of Mandre et al. (2009), (blue dashed line) for isothermal conditions $(\gamma=1)$ and (red dashed line) for adiabatic conditions $(\gamma=1.4)$.

We estimate the minimum centerline separation for the $V=4.05 \mathrm{~m} / \mathrm{s}$ video (Fig. $3 \mathrm{~d}$ ), as being $\simeq 480 \mathrm{~nm}$. For the highest impact velocity $V \simeq 6 \mathrm{~m} / \mathrm{s}$ we estimate the minimum height at only one dark and one bright fringe, i.e. $H^{*} \simeq 320 \mathrm{~nm}$.

Immediately following the contact, the trapped air-disc undergoes a rapid expansion, see Figs. $3(\mathrm{c}, \mathrm{d})$. For $V=2.43 \mathrm{~m} / \mathrm{s}$ the center has risen by a factor of $\sim 2$ in $5.6 \mu$ s after first contact, while for $V=4.05 \mathrm{~m} / \mathrm{s}$ it has grown by a factor of 3 in only $2.2 \mu \mathrm{s}$. This highlights the importance of our rapid imaging, to catch the detailed dynamics. At the end of this expansion, we see some overshoot and slight oscillations. For $V=2.43 \mathrm{~m} / \mathrm{s}$, in Fig. 3(c), the time from first contact to first maximum is $\Delta T=5.6 \mu \mathrm{s}$, while the duration until the subsequent expansion maximum after the last curve in Fig. 3(c), is $\Delta T=15.6 \mu \mathrm{s}$. For the highest impact velocity $(V=6.0 \mathrm{~m} / \mathrm{s})$ the expansion is faster and we can observe more oscillation cycles, with each subsequent expansion cycle taking longer, i.e. $\Delta T=0.4,3.4,4.4 \& 5.2 \mu \mathrm{s}$.

We integrate the air volume at first entrapment and after the maximum early expansion, from the video frames. This can be compared to the theory of Mandre et al. (2009), which gives the airfilm thickness at the start of compression as $H_{c} \sim R\left(\mu_{q} V /\left(R P_{o}\right)\right)^{1 / 2}=R S t^{2 / 3} \epsilon^{-1 / 2}$ and maximal compression at $H_{\text {min }} \sim R S t^{2 / 3} \epsilon^{(2-\gamma) /(2 \gamma-1)}=R S t^{2 / 3} \epsilon^{1 / 3}$. The ratio of these two gives the volume compression $\Omega_{b} / \Omega_{d i s c} \sim \epsilon^{-5 / 6}$, or incorporating our experimentally obtained empirical result $H^{*} \sim$ $\epsilon^{0.40}$ we get a slightly different powerlaw $\Omega_{b} / \Omega_{d i s c} \sim \epsilon^{-0.9}$. Figure 7 shows reasonable correspondence with this theory, with the ratio growing as large as a factor of $5(\Delta)$ for the largest $V$. However, comparing this expanded air volume to that contained in the final spherical bubble detached from the surface, additional volume increase is observed ( $\square$ in Fig. 7). This was also observed by Liu et al. (2013), while their lower frame-rate could not catch the earliest part of the expansion. With $d t=7 \mu$ s their images are essentially snapshots of the earliest evolution. We propose that the conical air-disc is still subject to significant dynamic pressure, due to the ongoing impact so soon after first contact. This pressure is released later as the disc pulls into a bubble, further increasing the volume ratios. We observe a total compression as large as a factor of 14 for the largest $V$.

The very large pressure produced just prior to the first contact, could potentially deform the solid locally. To probe possible effects on the air disc, we conducted a few separate experiments using a 


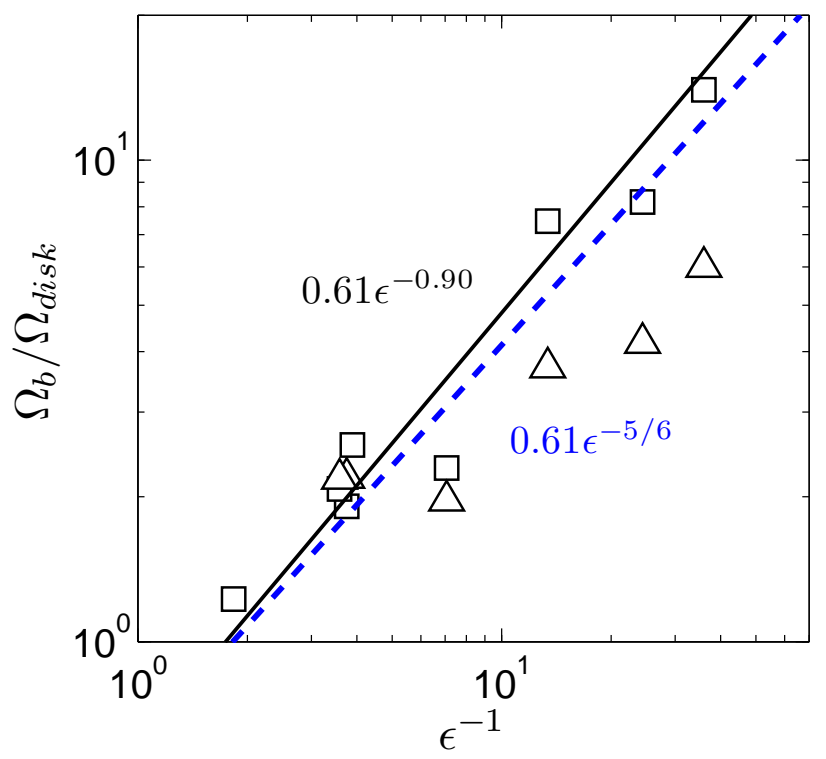

Figure 7. Compression of the air disc vs the impact parameter in Eq. (3.3). The compression of the air disc at the first contact along the ring $(\square)$ and after the first vertical expansion at the centerline $(\triangle)$. $\Omega_{\text {disc }}$ comes from integrating the thickness profile from the interferometry, whereas $\Omega_{b}$ is the volume of the final bubble, left behind after the contraction of the air disc.

much thinner, $150 \mu \mathrm{m}$-thick, glass slide (Menzel-Deckglacer), seeing no change in the thickness of the entrapped air disc.

\section{Discussion and conclusions}

Herein we have used the latest high-speed video technology for time-resolved interferometry to measure the lubricating air layer under an impacting drop, for higher velocities than previously possible. We have thereby characterized the dynamics well into the regime where the gas compressibility is significant. The results are overall in good agreement with theoretical models based on the lubricating air layer and inviscid drop dynamics (Mandre et al. (2009), Mandre \& Brenner (2012), Hicks \& Purvis (2010)). Our result for the size of the air-disc $L_{0}$ shows a perfect fit to the expression from Hicks \& Purvis (2013), without any adjustable constants.

Furthermore, our measurements of the air-layer thickness shed new light on the thermodynamics of the gas. The compression and entrapment of the air disc, occurs very rapidly and one can thereby expect non-equilibrium thermodynamics within the gas. Mandre et al. (2009) and Mani et al. (2010) used the ideal-gas equation of state,

$$
p=P_{0}\left(\frac{\rho}{\rho_{0}}\right)^{\gamma},
$$

to model the compression of the gas, where $p$ is the gas pressure, $\rho$ is the gas density, $P_{0}$ is the ambient pressure of the gas at density $\rho_{0}$. The value of $\gamma$ is chosen to be 1.4 for adiabatic, or 1 for isothermal gas compression. Later, Mandre \& Brenner (2012) argued that for highly thermally conducting substrates like metal or glass, the solid has a much larger thermal capacity than the gas layer and the time-scale of the impact is larger than that required for the gas temperature to become uniform. Therefore, one should use $\gamma=1$. Liu et al. (2013) also argued for the use of the isothermal $(\gamma=1)$ value in their study, estimating a typical conduction time of $30 \mathrm{~ns}$, for atmospheric gas density. We suggest that greatly increased gas density during the compression may increase this time significantly.

On the other hand, Hicks \& Purvis (2013) conducted a full thermodynamics analysis of the energy conservation in the gas and found that "the previous use of either an isothermal or an adiabatic equation of state is inappropriate in this regime". Hicks \& Purvis (2013) demonstrated large differences in the temperature profiles and in the density profiles at touchdown, between their compressible model and the earlier isothermal or adiabatic theories. They also showed another key difference between their compressible model and the earlier adiabatic case, i.e. the initial radius 
of the air pocket ( $L_{o}$ in our study) is independent of the compressibility in their full analysis; while they explain that $L_{o}$ will decrease as compressibility rises in the adiabatic case, as implied by Mandre et al. (2009).

Our comparison of the measured thickness of the air-layer with the theory of Mandre et al. (2009), shows that in the context of their simplified thermodynamic model, the adiabatic dynamics $(\gamma=1.4)$ gives a much better fit, than the isothermal dynamics, where the powerlaw should be much steeper, with an exponent of 1 . We find empirically that the best fit is $H^{*}=4.2 \epsilon^{0.40}$, with a steeper exponent than the theoretical prediction of $1 / 3$ in Mandre et al. (2009). To test these power-laws at even large values of the dynamic compression $\epsilon^{-1}$, will require future experiments at reduced ambient pressures.

Experiments at even higher impact velocities, than presented herein, would be of interest, but are challenging. This is especially true at the largest optical magnifications, owing to the random lateral drift of the center of the drop, which over a $2 \mathrm{~m}$ free-fall, is much larger than the 1-mm-wide field of view of the camera. For the largest impact heights, we need dozens of trials to catch any part of the disc, even for a reduced magnification of $2 \mu \mathrm{m} / \mathrm{px}$.

The leap in video frame-rate and pixel numbers of the camera used in our study (Crooks et al. (2013)), opens many other aspects of the drop impact to future detailed scrutiny. This includes the effects of reduced air pressure, Xu et al. (2005), or surface roughness, Latka et al. (2012). For example, for our drops of $D=2.6 \mathrm{~mm}$, impacting at $V=2.5 \mathrm{~m} / \mathrm{s}$, Mandre \& Brenner (2012) suggest that the air film can stay intact and the drop skate on it, if the surface roughness is smaller than $10 \mathrm{~nm}$. This technique is also ideal to study the breakup of the levitated films at the outer edge of the lamella, see Thoroddsen et al. (2010), Driscoll et al. (2010).

Research reported in this publication was supported by funding from King Abdullah University of Science and Technology (KAUST). We acknowledge anonymous referees for helpful suggestions.

\section{REFERENCES}

Boumhuis, W., van der Veen, R. C. A., Tran, T., Keij, D. L., Winkels, K. G., Peters, I. R., van der Meer, D., Sun, C., Snoeijer, J. H. \& Lohse, D. 2012 Maximal air bubble entrainment at liquid-drop impact. Phys. Rev. Lett. 109, 264501.

Chandra, S. \& Avedisian, C. T. 1991 On the collision of a droplet with a solid surface. Proc. R. Soc. Lond. A, 432, 13-41.

Crooks, J., Marsh, B., Turchetta, R., Taylor, K., Chan, W., Lahav, A. \& Fenigstein, A. 2013 Kirana: a solid-state megapixel uCMOS image sensor for ultrahigh speed imaging. Proc. SPIE, 8659, 865903.

De Ruiter, J., Lagraauw, R., van den Ende, D \& Mugele, F. 2014 Wettability-independent bouncing on flat surfaces mediated by thin air films. Nat. Phys. 11, 48-53.

De Ruiter, J., Oh, J. M., van den Ende, D. \& Mugele, F. 2012 Dynamics of collapse of air films in drop impact. Phys. Rev. Lett., 108, 074505.

Driscoll, M. M. \& NAgel, S. R. 2011 Ultrafast interference imaging of air in splashing dynamics. Phys. Rev. Lett., 107, 154502.

Driscoll, M. M., Stevens, C. S. \& Nagel, S. R. 2010 Thin film formation during splashing of viscous liquids. Phys. Rev. E, 82, 036302.

Duchemin, L. \& Josserand, C. 2011 Curvature singularity and film-skating during drop impact. Phys. Fluids 23, 091701.

Hicks, P. D., Ermanyuk, E. V., Gavrilov, N. V. \& Purvis, R. 2012 Air trapping at impact of a rigid sphere onto a liquid. J. Fluid Mech., 695, 310-320.

Hicks, P. D. \& Purvis, R. 2010 Air cushioning and bubble entrapment in three-dimensional droplet impacts. J. Fluid Mech., 649, 135-163.

Hicks, P. D. \& Purvis, R. 2013 Liquid-solid impacts with compressible gas cushioning. J. Fluid Mech., 735, 120-149.

Klaseboer, E., Manica, R. \& Chan, D. Y. C. 2014 Universal behavior of the initial stage of drop impact. Phys. Rev. Lett., 113, 194501.

Kolinski, J. M., Mahadevan, L. \& Rubinstein, S. M. 2014 Drops can bounce from perfectly hydrophilic surfaces. Euro. Phys. Lett. 108, 24001.

Kolinski, J. M., Rubinstein, S. M., Mandre, S., Brenner, M. P., Weitz, D. A. \& Mahadevan, L. 2012 Skating on a film of air: drops impacting on a surface. Phys. Rev. Lett., 108, 074503.

Korobkin, A. A., Ellis, A. S. \& Smith, F. T. 2008 Trapping of air in impact between a body and shallow water. J. Fluid Mech., 611, 365-394. 
Latka, A., Strandburg-Peshinin, A., Driscoll, M. M., Stevens, C. S. \& Nagel S. R. 2012. Creation of prompt and thin-sheet splashing by varying surface roughness or increasing air pressure, Phys. Rev. Lett., 109, 054501.

Lee, J. S., Weon, B. M., Je, J. H. \& FezzaA, K. 2012 How does an air film evolve into a bubble during drop impact? Phys. Rev. Lett. 109, 204501.

LiU, Y., TAN, P. \& XU, L. 2013 Compressible air entrapment in high-speed drop impacts on solid surfaces. J. Fluid Mech., 716, R9.

Mandre, S. \& Brenner, M. P. 2012 The mechanism of a splash on a dry solid surface. J. Fluid. Mech. 690, 148-172.

Mandre, S., Mani, M. \& Brenner, M. P. 2009 Precursors to splashing of liquid droplets on a solid surface. Phys. Rev. Lett. 102, 134502.

Mani, M., Mandre, S. \& Brenner, M. P. 2010 Events before droplet splashing on a solid surface. J. Fluid. Mech. 647, 163-185.

Richard, D., Clanet C. \& Quéré, D. 2002 Contact time of a bouncing drop. Nature, 417, 811.

Sмith, F. T., Li, L. \& Wu, G. X. 2003 Air cushioning with a lubrication/inviscid balance. J. Fluid Mech. 482, 291-318.

Thoraval, M. -J., Takehara, K., Etoh, T. G. \& Thoroddsen, S. T. 2013 Drop impact entrapment of bubble rings. J. Fluid Mech., 724, 234-258.

Thoroddsen, S. T., Eтoh, T. G., Takehara, K., Ootsuka, N. \& Hatsuki, Y. 2005 The air-bubble entrapped under a drop impacting on a solid surface. J. Fluid Mech., 545, 203-212.

Thoroddsen, S. T. \& Sakakibara, J. 1998 Evolution of the fingering pattern of an impacting drop. Phys. Fluids, 10, 1359-1373.

Thoroddsen, S. T., Takehara, K. \& Eтон, T. G. 2010 Bubble entrapment through topological change. Phys. Fluids, 22, 051701.

Thoroddsen, S. T., Takehara, K. \& Eтон, T. G. 2012 Micro-splashing by drop impacts. J. Fluid Mech., 706, 560-570.

Van Dam, D. B. \& Le Clerc, C. 2004 Experimental study of the impact of an ink-jet printed droplet on a solid substrate. Phys. Fluids, 16, 3403-3414.

van der Veen, R. C. A., Tran, T., Lohse, D. \& Sun, C. 2012 Direct measurements of air layer profiles under impacting droplets using high-speed color interferometry. Phys. Rev. E 85, 026315.

Wang, A.-B., Kuan, C.-C. \& Tsai, P.-H. 2013 Do we understand the bubble formation by a single drop impacting upon liquid surface?, Phys. Fluids, 25, 101702.

Xu, L., Zhang, W. W. \& Nagel, S. R. 2005 Drop splashing on a dry smooth surface. Phys. Rev. Lett., 94, 184505 . 